\title{
The Discrete Coagulation-Fragmentation Equations: Existence, Uniqueness, and Density Conservation
}

\author{
J. M. Ball ${ }^{1}$ and J. Carr ${ }^{1}$
}

Received October 25, 1989

\begin{abstract}
The discrete coagulation-fragmentation equation describes the kinetics of cluster growth in which clusters can coagulate via binary interactions to form larger clusters or fragment to form smaller ones. These models have many applications in pure and applied science ranging from cluster formation in galaxies to the kinetics of phase transformations in binary alloys. Our results relate to existence, uniqueness, density conservation and continuous dependence and they generalise the corresponding results in [ref. 2] for the Becker-Doring equations for which the processes are restricted to clusters gaining or shedding one particle. Examples are given which illustrate the role of the assumptions on the kinetic coefficients and show the rich set of analytic phenomena supported by the general discrete coagulation-fragmentation equations.
\end{abstract}

KEY WORDS: Existence theorems; admissibility; coagulation; fragmentation; clustering.

\section{INTRODUCTION}

In this paper we discuss the mathematical theory of a model for the dynamics of cluster growth. Such models arise in a wide variety of situations; examples include astrophysics, atmospheric physics, biology, colloidal chemistry, polymer science, and the kinetics of phase transitions in binary alloys. The basis for the models is that the system under consideration can be viewed as consisting of a large number of clusters that can coagulate to form larger clusters or fragment to form smaller ones. In the model analyzed in this paper the clusters are assumed to be discrete, that is, they consist of a finite number of smaller particles. The particles may be atoms, molecules, cells, etc., depending on the application.

\footnotetext{
${ }^{1}$ Department of Mathematics, Heriot-Watt University, Riccarton, Edinburgh EH14 4AS, Scotland, United Kingdom.
} 
If $c_{j}(t) \geqslant 0, j=1,2, \ldots$, denotes the expected number of clusters consisting of $j$ particles ( $j$-clusters) per unit volume, then the discrete coagulationfragmentation equations are

$$
\dot{c}_{j}=\frac{1}{2} \sum_{k=1}^{j-1}\left(a_{j-k, k} c_{j-k} c_{k}-b_{j-k, k} c_{j}\right)-\sum_{k=1}^{\infty}\left(a_{j, k} c_{j} c_{k}-b_{j, k} c_{j+k}\right)
$$

for $j=1,2, \ldots$. The coagulation rates $a_{j, k}$ and fragmentation rates $b_{j, k}$ are nonnegative constants with $a_{j, k}=a_{k, j}$ and $b_{j, k}=b_{k, j}$. In Eq. (1.1) the first two terms represent the rate of change of the $j$-cluster due to the coalescence of smaller clusters and the breakup of the $j$-cluster into smaller clusters. The final two terms represent the change due to coalescence of the $j$-cluster with other clusters and the breakup of larger clusters into $j$-clusters. For a derivation of this equation and its analogue in which the cluster size is a continuous variable see ref. 7. The model neglects (among other things) the geometrical location of clusters and spatial fluctuations in cluster density. For further information on these effects see refs. 5 and 6.

Since particles are neither created nor destroyed in the interactions described by (1.1), we expect the density $\rho=\sum_{j=1}^{\infty} j c_{j}(t)$ to be a conserved quantity. Mathematically, this is equivalent to

$$
\lim _{n \rightarrow \infty} \int_{0}^{t} \sum_{j=1}^{n} j \sum_{k=n-j+1}^{\infty} W_{j, k}(c(s)) d s=0
$$

where $W_{j, k}(c) \stackrel{\text { def }}{=} a_{j, k} c_{j} c_{k}-b_{j, k} c_{j+k}$. In certain circumstances, however, the density is not conserved. To illustrate this and other phenomena, we consider some special cases.

$\langle a\rangle$ Pure coagulation. Here $b_{j, k}=0$ for all $j$ and $k$. We further specialize to the following two idealized forms of coagulation kernel:

$$
\begin{aligned}
& a_{j, k}=j^{\alpha}+k^{\alpha} \\
& a_{j, k}=(j k)^{\alpha}
\end{aligned}
$$

where $\alpha>0$. The additive form (1.2) arises if we assume that binary interactions of clusters occur randomly with a rate proportional to the total effective surface area of the coagulating clusters. For compact clusters in $d$ dimensions $\alpha=1-d^{-1}$, but other values of $\alpha$ are also of interest. ${ }^{(8)}$ The multiplicative form (1.3) might apply to situations in which bond linking was the dominant mechanism. Note that, for the kernel (1.2), the rates for large-large and large-small interactions have the same order of magnitude (i.e., $a_{j, k} \cong a_{j, j}$ for large $j$ and small $k$ ), whereas for (1.3), large-large interactions dominate. 
If $\alpha>1 / 2$, then for the kernel (1.3) density conservation can break down in finite time. ${ }^{(8,9)}$ This is interpreted as the appearance of an infinite cluster or gel. For the kernel (1.2) we prove in Theorem 3.6 that if a solution exists, then density is conserved.

To gain some insight into the dependence of the rate of growth of clusters, we use a technique due to Leyvraz and Tschudi ${ }^{(10)}$ to relate solutions of (1.1) having different initial data. We first consider the kernel (1.2), so that (1.1) takes the form

$$
\dot{c}_{j}=\frac{1}{2} \sum_{k=1}^{j-1}\left[(j-k)^{\alpha}+k^{\alpha}\right] c_{j-k} c_{k}-\sum_{k=1}^{\infty}\left(j^{\alpha}+k^{\alpha}\right) c_{j} c_{k}
$$

Let $c_{j}^{1}$ be a solution of (1.4) with initial data $c_{j}^{1}(0)=\delta_{j, 1}$. For positive integers $n$, define $c^{n}(t)=\left(c_{j}^{n}(t)\right), j=1,2, \ldots$, by

$$
\begin{aligned}
& c_{n j}^{n}(t)=n^{-1} c_{j}^{1}\left(n^{\alpha-1} t\right) \\
& c_{r}^{n}(t)=0, \quad r \text { not a multiple of } n
\end{aligned}
$$

It is then easy to check that $c^{n}(t)$ is a solution of (1.4) with initial data given by $c_{j}^{n}(0)=n^{-1} \delta_{j, n}$. From (1.5) we see that the time scale for this class of solutions depends on the sign of $\alpha-1$. In fact, if $\alpha \leqslant 1$, we get global existence for the general initial value problem (with initial data having finite density), while if $\alpha>1$, we have nonexistence of global solutions.

For the kernel (1.3), let $c^{1}$ be the solution of (1.1) with initial data $c_{j}^{1}(0)=\delta_{j, 1}$. It is shown in ref. 10 that the appropriate scaling is

$$
c_{n j}^{n}(t)=n^{-1} c_{j}^{1}\left(n^{2 \alpha-1} t\right), \quad c_{r}^{n}(t)=0 \quad \text { otherwise }
$$

From (1.6), we see that $\alpha=1 / 2$ is the critical parameter value. Global solutions for the initial value problem exist for $\alpha \leqslant 1$ (see ref. 9 for a proof), but density conservation breaks down after a finite time if $\alpha>1 / 2$. It is interesting to note that if $\alpha>1$, we can still have global existence for this case. $^{(10)}$

(b) Pure fragmentation. Here $a_{j, k}=0$ for all $j, k$, so that (1.1) becomes linear. For any initial data with finite density, (1.1) has a densityconserving solution. However, for a large class of fragmentation coefficients (for example, $b_{j, k}=(j+k)^{\beta}, \beta>-1$ ), there are solutions with density $e^{t \lambda}$ for any $\lambda>0$; in particular, solutions need not be unique. These spurious solutions are not of physical interest, and this leads to the problem of finding a criterion for selecting the correct solution for the general equation (1.1).

(c) The Becker-Döring equations. Here $a_{j, k}=b_{j, k}=0$ if both $j$ and $k$ are greater than 1 . The mathematical theory of these equations has been 
studied in ref. 2 . In this case the density is always a conserved quantity. The asymptotic behavior of solutions is interesting both mathematically and for applications. Under certain hypotheses on the rate coefficients and the density of the initial data, we have that

$$
\rho=\sum_{j=1}^{\infty} j c_{j}(t)>\sum_{j=1}^{\infty} j \lim _{t \rightarrow \infty} c_{j}(t)=\rho_{s}
$$

The excess density $\rho-\rho_{s}$ corresponds to the formation of larger and larger clusters as $t \rightarrow \infty$, and may be interpreted as a transition from microscopic to macroscopic clusters. See also ref. 12 for an analysis of metastable solutions and refs. 3 and 14 for some technical refinements.

The aim of this paper is to obtain some of the fundamental results needed to extend the work on the Becker-Döring equation to more realistic models in which all interactions are allowed. The class of kinetic coefficients that we have in mind are $a_{j, k}=O\left(j^{\alpha}+k^{\alpha}\right)$ with $\alpha \leqslant 1$ and $b_{j, k}=a_{j, k} Q_{j} Q_{k}\left(Q_{j+k}\right)^{-1}$, where $Q_{j} \simeq z_{s}^{-j} \exp \left(-\lambda j^{p}\right)$ and $z_{s}, \lambda$, and $p$ are positive constants with $p<1$. (See Section 6 for a discussion.) In particular, we will not study situations in which coagulation can lead to density breakdown. In ref. 4 we use the theory developed here to study the asymptotic behavior of solutions.

Before outlining our results, we review what is known about the mathematical theory of solutions of (1.1). Spouge ${ }^{(15)}$ has proved existence under the assumption $a_{j, k}=o(j k)$ and a technical condition on $b_{j, k}$ which implies that it is bounded (see also ref. 9). White ${ }^{(17)}$ has proved existence under the assumptions $b_{j, k}=0, a_{j, k} \leqslant j^{\alpha}+k^{x}, \sum_{j=1}^{\infty} j^{m} c_{j}(0)<\infty$, where $0 \leqslant \alpha \leqslant 1$ and $m>\alpha$ is an integer. Aizenmann and $\mathrm{Bak}^{(1)}$ construct a complete mathematical theory for the continuous analogue of (1.1) for the case in which the kinetic rates are 1 . In particular, they single out the physical solution by using semigroup theory and choosing an appropriate domain for the linear operator associated with the fragmentation. Finally, Stewart ${ }^{(16)}$ has extended some of the results in this paper to the continuous analogue of (1.1).

Our results relate to existence, uniqueness, density conservation, and continuous dependence. The existence result (Theorem 2.4) generalizes the corresponding result in ref. 2 for the Becker-Döring equations and gives global existence when the initial data has finite density and $a_{j, k}=O(j+k)$. This is proved by taking the limit $N \rightarrow \infty$ of the system corresponding to (1.1) in which the maximum cluster size is $N$. Theorem 2.5 shows that solutions of (1.1) constructed in this way conserve density, thus excluding the nonphysical solutions mentioned earlier. It is also useful to have conditions under which all solutions conserve density. Such a result is given in 
Theorem 3.6. The condition on the fragmentation rates needed for this result has an interesting physical interpretation (see Section 6).

We give two uniqueness results; the first (Theorem 4.1) concerns all solutions and the second (Theorem 4.2) applies only to solutions which conserve density. We note that while Theorem 4.1 implies uniqueness for our motivating example (see Section 6), it would be desirable to extend our uniqueness results to a larger class of kinetic rates.

In Section 5 we study the differentiability of solutions and continuous dependence of solutions on initial data. Finally, in Section 6 we give a number of examples which illustrate the role of the assumptions on the kinetic coefficients.

\section{EXISTENCE OF DENSITY-CONSERVING SOLUTIONS}

We first introduce some notation. Let

$$
X=\left\{y=\left(y_{r}\right):\|y\|<\infty\right\}, \quad\|y\|=\sum_{r=1}^{\infty} r\left|y_{r}\right|
$$

$(X,\|\cdot\|)$ is a Banach space. We write $y \geqslant 0$ if $y_{r} \geqslant 0$ for each $r$ and set $X^{+}=\{y \in X: y \geqslant 0\}$.

As well as strong (norm) convergence in $X$, we will make use of weak* convergence: a sequence $y^{m}$ converges in the weak* sense to $y$ in $X$ (symbolically $y^{m} \stackrel{*}{*}_{y}$ ) if (i) $\sup _{m}\left\|y^{m}\right\|<\infty$ and (ii) $y_{r}^{m} \rightarrow y_{r}$ as $m \rightarrow \infty$ for each $r$.

Definition. Let $0<T \leqslant \infty$. A solution $c=\left(c_{j}\right)$ of $(1.1)$ on $[0, T)$ is a function $c:[0, T) \rightarrow X^{+}$such that:

(i) Each $c_{j}:[0, T) \rightarrow \mathbb{R}$ is continuous and $\sup _{t \in[0, T)}\|c(t)\|<\infty$.

(ii) For $j=1,2, \ldots$,

$$
\int_{0}^{t} \sum_{k=1}^{\infty} a_{j, k} c_{k}(s) d s<\infty, \quad \int_{0}^{t} \sum_{k=1}^{\infty} b_{j, k} c_{j+k}(s) d s<\infty
$$

for all $t \in[0, T)$.

(iii) For $j=1,2, \ldots$,

$$
c_{j}(t)=c_{j}(0)+\int_{0}^{t}\left[\frac{1}{2} \sum_{k=1}^{j-1} W_{j-k, k}(c(s))-\sum_{k=1}^{\infty} W_{j, k}(c(s))\right] d s
$$

for all $t \in[0, T)$. 
It follows easily from the above definition that if $c$ is a solution on $[0, T)$, then each $c_{r}$ is absolutely continuous, so that $c$ satisfies (1.1) for a.e. $t \in[0, T)$.

As in earlier work on similar equations, ${ }^{(11,13,15)}$ we prove existence of solutions by taking a limit of solutions of the finite-dimensional system

$$
\begin{gathered}
\dot{c}_{j}=\frac{1}{2} \sum_{k=1}^{j-1} W_{j-k, k}(c)-\sum_{k=1}^{n-j} W_{j, k}(c), \quad 1 \leqslant j \leqslant n \\
c_{j}(0) \geqslant 0, \quad 1 \leqslant j \leqslant n
\end{gathered}
$$

The following identity will be useful for finding bounds on solutions of $(2.1)$.

Lemma 2.1. Let $c$ be a solution of $(2.1)$ and let $\left(g_{j}\right)$ be a sequence of real numbers. Then for $1 \leqslant m \leqslant n$,

$$
\sum_{j=m}^{n} g_{j} \dot{c}_{j}=\frac{1}{2} \sum_{T_{1}}\left(g_{j+k}-g_{j}-g_{k}\right) W_{j, k}+\frac{1}{2} \sum_{T_{2}} g_{j+k} W_{j, k}+\sum_{T_{3}}\left(g_{j+k}-g_{k}\right) W_{j, k}
$$

where

$$
\begin{aligned}
& T_{1}=\{(j, k): j, k \geqslant m, j+k \leqslant n\} \\
& T_{2}=\{(j, k): m \leqslant j+k \leqslant n, j, k<m\} \\
& T_{3}=\{(j . k): 1 \leqslant j \leqslant m-1, k \geqslant m, j+k \leqslant n\}
\end{aligned}
$$

with the sums equal to zero if the associated region is empty. (See Fig. 1.)

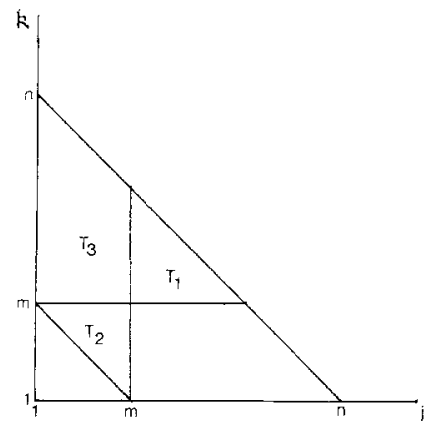

(a)

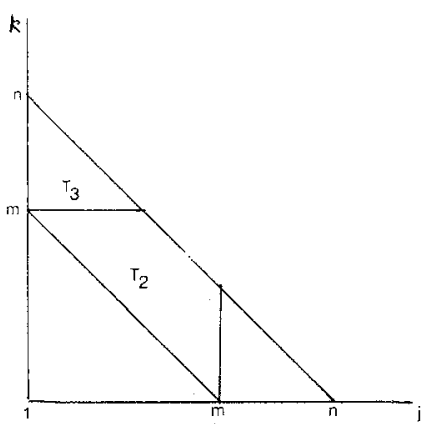

(b)

Fig. 1. Location of the region $T_{k}$ for the cases (a) $2 m<n$, (b) $2 m>n$. 
Proof. Let $T_{4}=\{(j, k): 1 \leqslant k \leqslant m-1, j \geqslant m, j+k \leqslant n\}$. Then the $T_{i}$, $i=1, \ldots, 4$, are disjoint, and $\bigcup_{i=1}^{4} T_{i}=\{(j, k): m \leqslant j+k \leqslant n\}$. Using the symmetry of the coagulation and fragmentation coefficients and (2.1), we have that

$$
\sum_{j=m}^{n} g_{j} \dot{c}_{j}=\frac{1}{2} \sum_{m \leqslant j+k \leqslant n} g_{j+k} W_{j, k}-\frac{1}{2} \sum_{j=m}^{n-1} g_{j} \sum_{k=m}^{n-j} W_{j, k}-\frac{1}{2} \sum_{k=m}^{n-1} g_{k} \sum_{j=1}^{n-k} W_{j, k}
$$

the last two terms being equal. The result follows from grouping the above terms into common regions in $j-k$ space.

Lemma 2.2. The system (2.1) has a unique solution for $t \geqslant 0$ with $c_{j}(t) \geqslant 0,1 \leqslant j \leqslant n$, and $\sum_{j=1}^{n} j c_{j}(t)=\sum_{j=1}^{n} j c_{j}(0)$.

Proof. The nonnegativity of each $c_{j}(t)$ may be proved in exactly the same way as the corresponding result in ref. 2 (see also ref. 15 for an alternative proof). The fact that $\sum_{j=1}^{n} j c_{j}(t)$ is a constant of the motion follows by setting $g_{j}=j$ in Lemma 2.1 , and the global existence follows from the bounds $0 \leqslant c_{j}(t) \leqslant j^{-1} \sum_{j=1}^{n} j c_{j}(0)$.

Lemma 2.3. Assume that $a_{j, k} \leqslant K_{0} j k$ for all $j, k \geqslant 1$, where $K_{0}$ is a constant. Let $c^{n}$ be a solution of $(2.1)$ and let $\rho^{n}(0)=\sum_{j=1}^{n} j c_{j}(0)$. Then

$$
\frac{d}{d t}\left\{e^{-t}\left[\sum_{j=m}^{n} j c_{j}^{n}(t)+2 m K_{0} \rho^{n}(0)^{2}\right]\right\} \leqslant 0
$$

for all $m \leqslant n, t \geqslant 0$.

Proof. By Lemma 2.1,

$$
\frac{d}{d t} \sum_{j=m}^{n} j c_{j}^{n}(t)=\frac{1}{2} \sum_{T_{2}}(j+k) W_{j, k}\left(c^{n}\right)+\sum_{T_{3}} j W_{j, k}\left(c^{n}\right)
$$

Hence

$$
\begin{aligned}
& \frac{d}{d t}\left\{e^{-t}\left[\sum_{j=m}^{n} j c_{j}^{n}(t)+2 m K_{0} \rho^{n}(0)^{2}\right]\right\} \\
& \quad=e^{-t}\left[\frac{1}{2} \sum_{T_{2}}(j+k) W_{j, k}+\sum_{T_{3}} j W_{j, k}-\sum_{j=m}^{n} j c_{j}^{n}(t)-2 m K_{0} \rho^{n}(0)^{2}\right] \\
& \quad \leqslant e^{-t}\left[\frac{1}{2} \sum_{T_{2}}(j+k) a_{j, k} c_{j}^{n} c_{k}^{n}+\sum_{T_{3}} j a_{j, k} c_{j}^{n} c_{k}^{n}-2 m K_{0} \rho^{n}(0)^{2}\right] \\
& \quad \leqslant K_{0} e^{-t}\left[\frac{1}{2} \sum_{T_{2}}(j+k) j k c_{j}^{n} c_{k}^{n}+\sum_{T_{3}} j^{2} k c_{j}^{n} c_{k}^{n}-2 m K_{0} \rho^{n}(0)^{2}\right]
\end{aligned}
$$


Now

$$
\sum_{T_{2}}(j+k) j k c_{j}^{n} c_{k}^{n} \leqslant 2 m \sum_{T_{2}} j k c_{j}^{n} c_{k}^{n} \leqslant 2 m \rho^{n}(0)^{2}
$$

and

$$
\sum_{T_{3}} j^{2} k c_{j}^{n} c_{k}^{n} \leqslant m \sum_{T_{3}} j k c_{j}^{n} c_{k}^{n} \leqslant m \rho^{n}(0)^{2}
$$

The result follows.

The following existence theorem generalizes to the full set of discrete coagulation-fragmentation equations the corresponding result in ref. 2 , Corollary 2.3, for the Becker-Döring equations. The proof follows a similar pattern, the main difficulty being to find appropriate generalizations of the a priori estimates. However, one difference is the use of Helly's theorem together with the preceding Lemma 2.3 to extract a convergent subsequence of approximating solutions; this simplifies the corresponding argument in ref. 2.

Theorem 2.4. Assume that $a_{j, k} \leqslant K(j+k)$, for all $j, k \geqslant 1$, where $K$ is a constant. Let $c_{0} \in X^{+}$. Then there exists a solution $c$ of $(1.1)$ on $[0, \infty)$ with $c(0)=c_{0}$.

Proof. Let $c^{n}(0)=\left(c_{01}, c_{02}, \ldots, c_{0 n}\right)$. By Lemma 2.2, the system (2.1) has a unique solution $c^{n}$ on $[0, \infty)$ with $c_{j}^{n}(t) \geqslant 0$ for $1 \leqslant j \leqslant n$ and

$$
\sum_{j=1}^{n} j c_{j}^{n}(t)=\sum_{j=1}^{n} j c_{j}(0) \quad \text { for all } t \geqslant 0
$$

We regard $c^{n}(t)$ as an element of $X^{+}$by defining $c_{j}^{n}(t)=0$ if $j>n$. Thus, $\left\|c^{n}(t)\right\| \leqslant\left\|c_{0}\right\|$ and $0 \leqslant c_{j}^{n}(t) \leqslant j^{-1}\left\|c_{0}\right\|$ for all $j$ and $n$. Let

$$
\vartheta_{m}^{n}(t)=e^{-t}\left[\sum_{j=m}^{n} j c_{j}^{n}(t)+2 m K_{0} \rho^{n}(0)^{2}\right]
$$

where $K_{0}=2 K$. By (2.3) and Lemma 2.3, for each fixed $m$, the functions $\vartheta_{m}^{n}(\cdot), n \geqslant m$, are of uniformly bounded variation on $[0, \infty)$. Hence, by Helly's theorem, there exists a subsequence, again denoted by $\vartheta_{m}^{n}$, such that $\vartheta_{m}^{n}(t) \rightarrow \vartheta_{m}(t)$ as $n \rightarrow \infty$ for each $t \geqslant 0$, for some function $\vartheta_{m}$ of bounded variation. Since

$$
c_{j}^{n}(t)=j^{-1} e^{t}\left[\vartheta_{j}^{n}(t)-\vartheta_{j+1}^{n}(t)\right]+2 j^{-1} K_{0} \rho^{n}(0)^{2}
$$

it follows that there exist a subsequence, which we continue to denote by $c^{n}$, and functions $c_{j}:[0, \infty) \rightarrow \mathbb{R}$, each of bounded variation on every 
compact subset of $[0, \infty)$, such that $c_{j}^{n}(t) \rightarrow c_{j}(t)$ as $n \rightarrow \infty$ for each $t \geqslant 0$. Clearly, $c_{j}(t) \geqslant 0$ and

$$
\sum_{j=1}^{\infty} j c_{j}(t) \leqslant\left\|c_{0}\right\| \quad \text { for all } \quad t \geqslant 0
$$

In order to pass to the limit in the integrated form of the $J$ th equation in (2.1), we will prove that for each $T \geqslant 0, \varepsilon>0$, and positive integer $J$, there exist $M>J$ and $N_{0}>3 M$ such that

$$
\int_{0}^{T}\left[p_{M}^{n}(t)+x_{M}^{n}(t)\right] d t<\varepsilon \quad \text { for all } n \geqslant N_{0}
$$

where

$$
x_{m}^{n} \stackrel{\text { def }}{=} \sum_{j=m}^{n} j c_{j}^{n}, \quad p_{m}^{n} \stackrel{\text { def }}{=} \sum_{j=1}^{m-1} \sum_{k=2 m}^{n-1} j b_{j, k} c_{j+k}^{n}
$$

Applying Lemma 2.1 with $g_{j}=j$, we obtain

$$
\dot{x}_{m}^{n}(t)=\sum_{T_{3}} j \dot{W}_{j, k}\left(c^{n}\right)+\frac{1}{2} \sum_{T_{2}}(j+k) W_{j, k}\left(c^{n}\right)
$$

Let $2 m<n$. To obtain an estimate on the terms in the sum over $T_{2}$ in (2.6), we apply Lemma 2.1 with

$$
g_{j}= \begin{cases}j & \text { for } \quad m \leqslant j \leqslant 2 m \\ 2 m & 2 m+1 \leqslant j \leqslant n\end{cases}
$$

Then, with an obvious notation,

$$
\begin{aligned}
& \sum_{j=m}^{2 m} j \dot{c}_{j}^{n}+2 m \sum_{j=2 m+1}^{n} \dot{c}_{j}^{n} \\
& \quad=\frac{1}{2} \sum_{T_{1}} \mu_{j, k} W_{j, k}\left(c^{n}\right)+\frac{1}{2} \sum_{T_{2}}(j+k) W_{j, k}\left(c^{n}\right)+\sum_{T_{3}} \lambda_{j, k} W_{j, k}\left(c^{n}\right)
\end{aligned}
$$

For $(j, k) \in T_{1}$ we have that $\mu_{j, k}=\mu_{k, j}$ and

$$
\mu_{j, k}= \begin{cases}2 m-(j+k) & \text { for } j, k \leqslant 2 m \\ -j & \text { for } j \leqslant 2 m, \quad k>2 m \\ -2 m & \text { for } j>2 m, \quad k>2 m\end{cases}
$$


We note that

$$
0 \leqslant-\mu_{j, k} \leqslant 2 m \quad \text { for all } \quad(j, k) \in T_{1}
$$

For $(j, k) \in T_{3}$ we have that

$$
\lambda_{j, k}= \begin{cases}0 & \text { for } k \geqslant 2 m \\ j & \text { for } j+k \leqslant 2 m \\ 2 m-k & \text { for } j+k \geqslant 2 m+1, \quad k<2 m\end{cases}
$$

Furthermore,

$$
0 \leqslant \lambda_{j, k} \quad \text { and } \quad 0 \leqslant j-\lambda_{j, k} \quad \text { for all } \quad(j, k) \in T_{3}
$$

From (2.6)-(2.7), we obtain for $2 m<n$,

$$
\begin{aligned}
x_{m}^{n}(t)= & x_{m}^{n}(0)+q_{m}^{n}(t)-q_{m}^{n}(0) \\
& +\int_{0}^{t}\left[\sum_{T_{3}}\left(j-\lambda_{j, k}\right)-\sum_{T_{1}} \mu_{j, k}\right] W_{j, k}\left(c^{n}(s)\right) d s
\end{aligned}
$$

where

$$
q_{m}^{n} \stackrel{\text { def }}{=} \sum_{j=m}^{2 m} j c_{j}^{n}+2 m \sum_{j=2 m+1}^{n} c_{j}^{n}
$$

Since by (2.3), (2.4),

$$
\left|\sum_{j=L}^{\infty}\left[c_{j}^{n}(t)-c_{j}(t)\right]\right| \leqslant L^{-1} \sum_{j=L}^{\infty} j\left[c_{j}^{n}(t)+c_{j}(t)\right] \leqslant 2 L^{-1}\left\|c_{0}\right\|
$$

we deduce that for each $m$ and all $t \geqslant 0$,

$$
\lim _{n \rightarrow \infty} q_{m}^{n}(t)=\sum_{j=m}^{2 m} j c_{j}(t)+2 m \sum_{j=2 m+1}^{\infty} c_{j}(t) \stackrel{\text { def }}{=} q_{m}(t)
$$

Let $T>0$. Then $0 \leqslant q_{m}(t) \leqslant \sum_{j=m}^{\infty} j c_{j}(t)$, so that

$$
\lim _{m \rightarrow \infty} q_{m}(t)=0, \quad\left|q_{m}(t)\right| \leqslant \text { const } \quad \text { for all } t \in[0, T]
$$

Thus, given $\varepsilon>0$, there exists $M>J$ such that

$$
\int_{0}^{T} q_{M}(t) d t<\varepsilon \quad \text { and } \quad x_{M}^{n}(0)=\sum_{j=M}^{n} j c_{0 j}<\varepsilon / T \quad \text { for all } \quad n \geqslant M
$$


By (2.11), (2.12), there exists $N_{0}>3 M$ such that for $n \geqslant N_{0}$,

$$
\int_{0}^{t} q_{M}^{n}(s) d s<2 \varepsilon \quad \text { for all } t \in[0, T]
$$

Returning to (2.10) and using (2.8), (2.9), we have that for $t \in[0, T]$, $n \geqslant N_{0}$,

$$
\begin{aligned}
x_{M}^{n}(t) & +\int_{0}^{t} p^{n}(s) d s \\
& \leqslant \varepsilon / T+q_{M}^{n}(t)+\int_{0}^{t}\left(\sum_{T_{3}} j+2 M \sum_{T_{1}}\right) a_{j, k} c_{j}^{n}(s) c_{k}^{n}(s) d s
\end{aligned}
$$

From (2.13) we obtain for $t \in[0, T], n \geqslant N_{0}$,

$$
\begin{aligned}
& \int_{0}^{t} x_{M}^{n}(s) d s+\int_{0}^{t} \int_{0}^{s} p^{n}(\tau) d \tau d s \\
& \quad \leqslant 3 \varepsilon+\int_{0}^{t} \int_{0}^{s}\left(\sum_{T_{3}} j+2 M \sum_{T_{1}}\right) a_{j, k} c_{j}^{n}(\tau) c_{k}^{n}(\tau) d \tau d s
\end{aligned}
$$

Now

$$
\begin{aligned}
\sum_{T_{3}} j a_{j, k} c_{j}^{n} c_{k}^{n} & \leqslant K \sum_{j=1}^{M-1} j c_{j}^{n} \sum_{k=M}^{n-j}(j+k) c_{k}^{n} \\
& \leqslant 2 K \sum_{j=1}^{M-1} j c_{j}^{n} \sum_{k=M}^{n-j} k c_{k}^{n} \\
& \leqslant 2 K\left\|c_{0}\right\| x_{M}^{n}
\end{aligned}
$$

and

$$
2 M \sum_{T_{1}} a_{j, k} c_{j}^{n} c_{k}^{n} \leqslant 2 K M \sum_{j=M}^{n} \sum_{k=M}^{n}(j+k) c_{j}^{n} c_{k}^{n} \leqslant 4 K\left\|c_{0}\right\| x_{M}^{n}
$$

Using (2.16), (2.17) in (2.15) and applying Gronwall's inequality, it follows that for all $t \in[0, T], n \geqslant N_{0}$,

$$
\int_{0}^{t} x_{M}^{n}(s) d s+\int_{0}^{t} \int_{0}^{s} p^{n}(\tau) d \tau d s \leqslant K_{1} \varepsilon
$$

where $K_{1}$ is a constant depending only on $K, T$, and $\left\|c_{0}\right\|$. Since

$$
\int_{0}^{t} \int_{0}^{s} p^{n}(\tau) d \tau d s=\int_{0}^{t}(t-s) p^{n}(s) d s
$$


it follows from (2.18) that for all $n \geqslant N_{0}$

$$
\int_{0}^{T / 2} x_{M}^{n}(s) d s+\frac{T}{2} \int_{0}^{T / 2} p^{n}(s) d s \leqslant K_{1} \varepsilon
$$

With an appropriate rescaling of $T, \varepsilon$, this gives (2.5).

From the pointwise convergence of $c_{j}^{n}(t)$ and (2.5) we deduce that

$$
\int_{0}^{T}\left[\sum_{j=1}^{M-1} \sum_{k=2 M}^{\infty} j b_{j, k} c_{j+k}^{n}(s)+\sum_{j=M}^{\infty} j c_{j}(s)\right] d s \leqslant \varepsilon
$$

In particular, since $M>J$,

$$
\int_{0}^{T} \sum_{k=1}^{\infty} a_{J, k} c_{k}^{n}(s) d s<\infty, \quad \int_{0}^{T} \sum_{k=1}^{\infty} b_{J, k} c_{J+k}^{n}(s) d s<\infty
$$

For any $l>2 M, n>J+l, t \in[0, T]$ we have from (2.1), (2.5) that

$$
\begin{aligned}
& \left|c_{J}^{n}(t)-c_{J}(0)-\int_{0}^{t}\left[\frac{1}{2} \sum_{k=1}^{J-1} W_{J-k, k}\left(c^{n}(s)\right)-\sum_{k=1}^{l} W_{J, k}(c(s))\right] d s\right| \\
& \leqslant\left(2 K\left\|c_{0}\right\|+1\right) \varepsilon
\end{aligned}
$$

Letting $n \rightarrow \infty$ and then $l \rightarrow \infty$ in (2.21), we deduce by (2.20) and the arbitrariness of $\varepsilon$ that

$$
c_{J}(t)=c_{J}(0)+\int_{0}^{t}\left[\frac{1}{2} \sum_{k=1}^{J-1} W_{J-k, k}(c(s))-\sum_{k=1}^{\infty} W_{J, k}(c(s))\right] d s
$$

for all $t \geqslant 0$ and each $J$, as required. In particular, each $c_{J}$ is continuous on $[0, \infty)$.

In general, even if $a_{j, k} \leqslant K(j+k)$, solutions of (1.1) do not conserve density. The next result shows that for $a_{j, k} \leqslant K(j+k)$, the solution constructed in Theorem 2.4 conserves density.

Theorem 2.5. Assume that $a_{j, k} \leqslant K(j+k)$ and that $c$ is the solution constructed in Theorem 2.4. Then for all $t \geqslant 0$,

$$
\sum_{j=1}^{\infty} j \mathcal{c}_{j}(t)=\sum_{j=1}^{\infty} j c_{j}(0)
$$

Proof. Let $c^{n}$ be the solution to $(2.1)$ with $c^{n}(0)=c_{j}(0), 1 \leqslant j \leqslant n$. Then, writing $n$ for the subsequence $n_{k}$, we have that $c_{j}^{n}(t) \rightarrow c_{j}(t)$ as $n \rightarrow \infty$ for each $j$ and all $t \geqslant 0$. We use various relations derived in the 
proof of Theorem 2.4. In particular, from (2.7)-(2.10), (2.16) and (2.17) we obtain for $t \geqslant 0$

$$
x_{m}^{n}(t) \leqslant x_{m}^{n}(0)+q_{m}^{n}(t)+K_{2} \int_{0}^{t} x_{m}^{n}(s) d s
$$

where $K_{2}$ is a constant. Fix $t \geqslant 0$ and let $\varepsilon>0$. By (2.18) and

$$
\lim _{n \rightarrow \infty} q_{m}^{n}(t)=q_{m}(t), \quad \lim _{m \rightarrow \infty} q_{m}(t)=0, \quad x_{m}^{n}(0)=\sum_{j=m}^{n} j c_{j}(0), \quad c(0) \in X^{+}
$$

there exist $M$ and $N_{1}>M$, depending on $t$, such that

$$
\left|q_{M}^{n}(t)\right|<\varepsilon, \quad\left|x_{M}^{n}(0)\right|<\varepsilon, \quad \int_{0}^{t} x_{M}^{n}(s) d s<\varepsilon \quad \text { for all } n \geqslant N_{1}
$$

Using this in (2.23) gives

$$
x_{M}^{n}(t) \leqslant K_{3} \varepsilon \quad \text { for } n \geqslant N_{1}
$$

where $K_{3}$ is a constant. Thus, also

$$
\sum_{j=M}^{\infty} j c_{j}(t) \leqslant K_{3} \varepsilon
$$

Writing $c_{j}^{n}(t)=0$ for $j>n$, we deduce from (2.25)-(2.26) that for $n \geqslant N_{1}$

$$
\left|\sum_{j=1}^{\infty} j\left[c_{j}^{n}(t)-c_{j}(t)\right]\right| \leqslant\left|\sum_{j=1}^{M-1} j\left[c_{j}^{n}(t)-c_{j}(t)\right]\right|+2 K_{3} \varepsilon
$$

Since $\sum_{j=1}^{\infty} j c_{j}^{n}(t)=\sum_{j=1}^{\infty} j c_{j}(0)$, letting $n \rightarrow \infty$ in (2.27) gives

$$
\left|\sum_{j=1}^{\infty} j\left[c_{j}(0)-c_{j}(t)\right]\right| \leqslant 2 K_{3} \varepsilon
$$

and the result follows.

Corollary 2.6. Let the hypotheses of Theorem 2.5 hold, and denote by $c^{n_{k}}$ the corresponding pointwise convergent subsequence of solutions to (2.1). Then $c^{n_{k}}(t) \rightarrow c(t)$ in $X$ uniformly on compact subsets of $[0, \infty)$.

Proof. We again write $n$ for the subsequence $n_{k}$. We first prove that, for each $j, c_{j}^{n}(t) \rightarrow c_{j}(t)$ uniformly on compact subsets of $[0, \infty)$. For this it is clearly sufficient to show that for each $m>1$,

$$
y_{m}^{n}(t) \stackrel{\text { def }}{=} e^{-i}\left[\rho^{n}(0)-\sum_{j=1}^{m-1} j c_{j}^{n}(t)+4 m K \rho^{n}(0)^{2}\right]
$$


converges to

$$
y_{m}(t) \stackrel{\text { def }}{=} e^{-t}\left[\rho(0)-\sum_{j=1}^{m-1} j c_{j}(t)+4 m K \rho(0)^{2}\right]
$$

uniformly on compact subsets of $[0, T)$, where $\rho(0) \stackrel{\text { def }}{=} \sum_{j=1}^{\infty} j c_{j}(0)$. But this follows from the pointwise convergence of $y_{m}^{n}(t)$ to the continuous function $y_{m}(t)$ and the fact that by Lemmas $2.2,2.3$,

$$
\frac{d}{d t} y_{m}^{n}(t) \leqslant 0, \quad t \in[0, T), \quad n \geqslant m
$$

Let $I \subset[0, \infty)$ be compact and $t_{n} \rightarrow t$ in $I$. By Lemma 2.2 and Theorem 2.5

$$
\lim _{n \rightarrow \infty}\left\|c^{n}\left(t_{n}\right)\right\|=\lim _{n \rightarrow \infty}\left\|c\left(t_{n}\right)\right\|=\|c(t)\|
$$

Applying Lemma 2.7 below, we deduce that as $n \rightarrow \infty, c^{n}\left(t_{n}\right) \rightarrow c(t)$, $c\left(t_{n}\right) \rightarrow c(t)$ in $X$. Hence $c^{n} \rightarrow c$ in $C(I, X)$, as required.

Lemma 2.7. (Cf. ref. 2, Lemma 3.3). If $y^{n} \stackrel{*}{\rightarrow}$ in $X$ and $\left\|y^{n}\right\| \rightarrow\|y\|$, then $y^{n} \rightarrow y$ in $X$.

\section{CONDITIONS UNDER WHICH ALL SOLUTIONS CONSERVE DENSITY}

We first give an easily proved identity valid for solutions of (1.1) similar to the identity given in Lemma 2.1. The reader is encouraged to sketch the analogue of Fig. 1 for the regions $R_{k}$.

Lemma 3.1. Let $c$ be a solution of $(1.1)$ on $[0, T)$ and let $\left(g_{j}\right)$ be a sequence. Then for $1 \leqslant m \leqslant n$ and $0 \leqslant t_{1}<t_{2}<T$,

$$
\begin{aligned}
\sum_{j=m}^{n} g_{j}\left[c_{j}\left(t_{2}\right)-c_{j}\left(t_{1}\right)\right]= & \int_{t_{1}}^{t_{2}}\left[\frac{1}{2} \sum_{R_{1}}\left(g_{j+k}-g_{j}-g_{k}\right)\right. \\
& \left.+\frac{1}{2} \sum_{R_{2}} g_{j+k}+\sum_{R_{3}}\left(g_{j+k}-g_{k}\right)-\sum_{R_{4}} g_{j}\right] W_{j, k}(c(s)) d s
\end{aligned}
$$

where

$$
\begin{aligned}
& R_{1}=\{(j, k): j, k \geqslant m, j+k \leqslant n\} \\
& R_{2}=\{(j, k): m \leqslant j+k \leqslant n, j, k<m\} \\
& R_{3}=\{(j, k): 1 \leqslant j \leqslant m-1, k \geqslant m, j+k \leqslant n\} \\
& R_{4}=\{(j, k): m \leqslant j \leqslant n, j+k>n\}
\end{aligned}
$$


with the sums equal to zero if the associated region is empty. (Note that $R_{2}$ and $R_{3}$ are empty if $m=1$.)

Setting $g_{j}=j$ and $m=1$ in Lemma 3.1 , to prove that $\sum_{j=1}^{\infty} j c_{j}(t)$ is a conserved quantity, it is sufficient to prove that

$$
\lim _{n \rightarrow \infty} \int_{0}^{t} \sum_{j=1}^{n} j \sum_{k=n-j+1}^{\infty} W_{j, k}(c(s)) d s=0
$$

Considering, for example, the case $a_{j, k}=j+k, b_{j, k}=0$, it is clear that we will require more information on the solution in order to prove (3.2). The basic plan is as follows.

(a) Taking $g_{j}=1$ in Lemma 3.1 and letting $n \rightarrow \infty$, obtain an identity of the form

$$
\sum_{j=m}^{\infty}\left[c_{j}\left(t_{2}\right)-c_{j}\left(t_{1}\right)\right]=\int_{t_{1}}^{t_{2}} D_{m}(c(s)) d s
$$

(b) Since $c \in X^{+}$, for each $t, m \sum_{j=m}^{\infty} c_{j}(t) \leqslant \sum_{j=m}^{\infty} j c_{j}(t) \rightarrow 0$ as $m \rightarrow \infty$. Thus, from (3.3),

$$
\lim _{m \rightarrow \infty} m \int_{t_{1}}^{t_{2}} D_{m}(c(s)) d s=0
$$

The idea is to exploit the extra information contained in (3.4) to prove (3.2).

Taking $g_{j}=1$ in Lemma 3.1 and letting $n \rightarrow \infty$, we formally obtain

$$
\begin{aligned}
& \sum_{j=m}^{\infty}\left[c_{j}\left(t_{2}\right)-c_{j}\left(t_{1}\right)\right] \\
& =\frac{1}{2} \int_{t_{1}}^{t_{2}}\left[-\sum_{j=m}^{\infty} \sum_{k=m}^{\infty} W_{j, k}(c(s))+\sum_{j=1}^{m-1} \sum_{k=m-j}^{m-1} W_{j, k}(c(s))\right] d s
\end{aligned}
$$

In order to execute our plan, we will need to let $m \rightarrow \infty$ in (3.5) and to be able to manipulate the resulting double series. Thus, we will require that

$$
\begin{array}{r}
\int_{t_{1}}^{t_{2}} \sum_{j, k=1}^{\infty} a_{j, k} c_{j}(s) c_{k}(s) d s<\infty \\
\int_{t_{1}}^{t_{2}} \sum_{j, k=1}^{\infty} b_{j, k} c_{j+k}(s) d s<\infty
\end{array}
$$

This need not be the case even if (3.4) holds. 
Example 3.2. Let $a_{j, k}=(j k)^{3}(j+k)^{-2}, b_{j, k}=j+k, c_{j}=j^{-3}$. Then $c=\left(c_{j}\right) \in X^{+}$and $W_{j, k}(c)=0$ for all $j, k$. Also,

$$
\sum_{k=1}^{\infty} a_{j, k} c_{j} c_{k}=\sum_{k=1}^{\infty} b_{j, k} c_{j+k}=\sum_{k=1}^{\infty}(j+k)^{-2}<\infty
$$

so that $c$ is a solution of (1.1). In this case $D_{m}=0$. However, the double sums in (3.6)-(3.7) are infinite.

Lemma 3.3. Let $c$ be a solution of (1.1) on $[0, T)$ and let $0 \leqslant t_{1}<$ $t_{2}<T$. Suppose that either (3.6) or (3.7) holds. Then (3.5)-(3.7) hold and

$$
\lim _{m \rightarrow \infty} m \int_{t_{1}}^{t_{2}}\left(\sum_{j=m}^{\infty} \sum_{k=m}^{\infty}-\sum_{j=1}^{m-1} \sum_{k=m-j}^{m-1}\right) W_{j, k}(c(s)) d s=0
$$

Proof. Setting $g_{j}=1$ and $m=1$ in Lemma 3.1 gives

$$
\sum_{j=1}^{n}\left[c_{j}\left(t_{2}\right)-c_{j}\left(t_{1}\right)\right]=-\int_{t_{1}}^{t_{2}}\left(\frac{1}{2} \sum_{j+k \leqslant n}+\sum_{j=1}^{n} \sum_{k=n-j+1}^{\infty}\right) W_{j, k}(c(s)) d s
$$

Since $c \in X^{+}$, the right-hand side of (3.9) is bounded independently of $n$. Hence, if either (3.6) or (3.7) holds, so does the other. Letting $n \rightarrow \infty$ in (3.9) and using the dominated convergence theorem gives (3.5) for $m=1$; the case of general $m$ follows from adding on a finite sum. Finally, since $c \in X^{+}$, for fixed $t$ we have that

$$
m \sum_{j=m}^{\infty} c_{j}(t) \leqslant \sum_{j=m}^{\infty} j c_{j}(t) \rightarrow 0 \quad \text { as } \quad m \rightarrow \infty
$$

Combining this with (3.5) proves (3.8).

The following easily proved proposition gives some examples of kinetic coefficients which satisfy either (3.6) or (3.7).

Proposition 3.4. Let $c$ be a solution of (1.1) on $[0, T)$. Let $\left(r_{j}\right)$ be a nonnegative sequence and let $\alpha_{j, k}$ satisfy $\alpha_{j, k} \geqslant 0$ for all $j, k$ and $\alpha_{j, k} \leqslant K(j+k)$ for $j \geqslant n_{0}$ and $k \geqslant n_{0}$, where $K, n_{0}$ are constants. Then (3.6) and (3.7) hold in the following cases:

(i) $a_{j, k}=r_{j}+r_{k}+\alpha_{j, k}$.

(ii) $a_{j, k}=r_{j} r_{k}+\alpha_{j, k}$.

(iii) $b_{j, k} \equiv 0$ for all $j, k$ with $j \geqslant n_{0}$ and $k \geqslant n_{0}$.

We use below the following notation: if $r$ is an integer, then $h(r)=[(r+1) / 2]$, the integer part of $(r+1) / 2$. This notation is used in 
sums over $j, k$ space with $j+k=r$ up to the diagonal $j=k$. In practice the reader can think of $h(r)$ as equalling $r / 2$.

We show that all solutions of (1.1) conserve density under the following conditions (here $K$ and $n_{0}$ are constants):

(H1) $a_{j, k}=r_{j}+r_{k}+\alpha_{j, k}$, where $\left(r_{j}\right)$ is a nonnegative sequence and $\alpha_{j, k} \geqslant 0$ for all $j, k$, and $\alpha_{j, k} \leqslant K(j+k)$ for all $j, k \geqslant n_{0}$.

(H2) $\sum_{j=n_{0}}^{h(r)} j b_{r-j, j} \leqslant K r$ for all $r \geqslant 2 n_{0}$.

Note that if $a_{j, k}=b_{j, k}=0$ when both $j, k \geqslant n_{0}$, then (H1)-(H2) are trivially satisfied.

To prove conservation, we combine (3.8) with various estimates on sums of coagulation and fragmentation terms.

Lemma 3.5. Assume (H1)-(H2). Let $c$ be a solution of (1.1) on $[0, T)$ and let $t \in[0, T)$. Then if $\bar{W}_{j, k}(c(s))=a_{j, k} c_{j}(s) c_{k}(s)$ or $\bar{W}_{j, k}(c(s))=$ $b_{j, k} c_{j+k}(s)$

$$
\begin{array}{r}
\lim _{n \rightarrow \infty} \int_{0}^{t} \sum_{j=1}^{n} \sum_{k=h(n)}^{\infty} j \bar{W}_{j, k}(c(s)) d s=0 \\
\lim _{n \rightarrow \infty} n \int_{0}^{t} \sum_{j=n}^{\infty} \sum_{k=n}^{\infty} \bar{W}_{j, k}(c(s)) d s=0 \\
\lim _{n \rightarrow \infty} \int_{0}^{t} \sum_{T}(n-j) \bar{W}_{j, k}(c(s)) d s=0
\end{array}
$$

where

$$
T=\{(j, k): j+k \geqslant n, k \leqslant h(n), j \leqslant n-1\}
$$

Proof. We first make some observations which restrict the regions of summation in (3.11) and (3.13). From the definition of a solution,

$$
\lim _{n \rightarrow \infty} \int_{0}^{t} \sum_{j=1}^{n_{0}-1} \sum_{k=h(n)}^{\infty} \bar{W}_{j, k}(c(s)) d s=0
$$

Thus, to prove (3.11), we need only consider $j \geqslant n_{0}$. Also, set $T^{\prime}=\left\{(j, k) \in T: k \leqslant n_{0}-1\right\}$. Then

$$
\int_{0}^{t} \sum_{T^{\prime}}(n-j) \bar{W}_{j, k}(c(s)) d s \leqslant n_{0} \int_{0}^{t} \sum_{j=n-n_{0}}^{n} \sum_{k=1}^{n_{0}-1} \bar{W}_{j, k}(c(s)) d s \rightarrow 0
$$

as $n \rightarrow \infty$, by the definition of a solution. Thus, to prove (3.13), we need only sum over the region $R=\left\{(j, k) \in T: k \geqslant n_{0}\right\}$. 
We estimate the coagulation and fragmentation terms in (3.11)-(3.13) separately. Let

$$
u_{n}=\sum_{j=n_{0}}^{n} \sum_{k=h(n)}^{\infty} j a_{j, k} c_{j} c_{k}, \quad v_{n}=n \sum_{j=n}^{\infty} \sum_{k=n}^{\infty} a_{j, k} c_{j} c_{k}
$$

Using the assumptions on $a_{j, k}$, for $n$ sufficiently large,

$$
\begin{aligned}
u_{n} \leqslant & \sum_{j=n_{0}}^{\infty} j c_{j}\left(3 K \sum_{k=h(n)}^{\infty} k c_{k}+\sum_{k=h(n)}^{\infty} r_{k} c_{k}\right) \\
& +2 \sum_{j=n_{0}}^{n} r_{j} c_{j} \sum_{k=h(n)}^{\infty} k c_{k} \\
v_{n} \leqslant & 2 \sum_{k=n}^{\infty} k c_{k}\left(\sum_{j=n}^{\infty} r_{j} c_{j}+K \sum_{j=n}^{\infty} j c_{j}\right)
\end{aligned}
$$

From the definition of a solution, $\sup _{s \in[0, t]}\|c(s)\|<\infty$ and $\int_{0}^{t} \sum_{j=1}^{\infty} r_{j} c_{j}(s) d s<\infty$, so that as $n \rightarrow \infty$,

$$
\begin{aligned}
\int_{0}^{t} & \sum_{j=n}^{\infty} j c_{j}(s) d s, \int_{0}^{t} \sum_{j=n}^{\infty} r_{j} c_{j}(s) d s, \\
& \left.\int_{0}^{t}\left[\sum_{j=n}^{\infty} j c_{j}(s) \sum_{k=1}^{\infty} r_{k} c_{k}(s)\right)\right] d s \rightarrow 0
\end{aligned}
$$

Using (3.14)-(3.16), $\int_{0}^{t} u_{n}(s) d s, \int_{0}^{t} v_{n}(s) d s \rightarrow 0$ as $n \rightarrow \infty$, which proves (3.11)-(3.12) for the coagulation terms. Now

$$
\begin{aligned}
w_{n} & =\sum_{R}(n-j) a_{j, k} c_{j} c_{k} \\
& \leqslant \sum_{j=h(n)}^{n-1} \sum_{k=n-j}^{h(n)}(n-j)\left[K(j+k)+r_{j}+r_{k}\right] c_{j} c_{k}
\end{aligned}
$$

In the above sum, $n-j \leqslant j$ and $n-j \leqslant k$. Thus,

$$
w_{n} \leqslant \sum_{j=h(n)}^{n} j c_{j}\left(2 K \sum_{k=1}^{h(n)} k c_{k}+\sum_{k=1}^{h(n)} r_{k} c_{k}\right)+\sum_{j=h(n)}^{n} r_{j} c_{j} \sum_{k=1}^{h(n)} k c_{k}
$$

so that by (3.16), $\int_{0}^{t} w_{n}(s) d s \rightarrow 0$ as $n \rightarrow \infty$. This proves (3.13) for the coagulation terms.

We now estimate the fragmentation terms. Let

$$
\bar{v}_{n}=n \sum_{j=n}^{\infty} \sum_{k=n}^{\infty} b_{j, k} c_{j+k}=n \sum_{r=2 n}^{\infty} c_{r} \sum_{j=n}^{r-n} b_{r-j, j}
$$


For $r \geqslant 2 n$,

$$
n \sum_{j=n}^{r-n} b_{r-j, j} \leqslant 2 n \sum_{j=n}^{h(r)} b_{r-j, j} \leqslant 2 \sum_{j=n}^{h(r)} j b_{r-j, j}
$$

so that by (H2), $\bar{v}_{n} \leqslant 2 K \sum_{r=2 n}^{\infty} r c_{r}$ for $n \geqslant n_{0}$, which proves (3.12).

Using (3.12) with $n$ replaced by $h(n)$, we see that to prove (3.11) we need only estimate

$$
\sum_{j=n_{0}}^{h(n)} \sum_{k=h(n)}^{\infty} j b_{j, k} c_{j+k}=\sum_{r=h(n)+n_{0}}^{\infty} c_{r} \sum_{j=n_{0}}^{q} j b_{r-j, j}
$$

where $q=\min (h(n), r-h(n)) \leqslant h(r)$. Integrating (3.18) and using (H2), we obtain (3.11).

Finally,

$$
\sum_{R}(n-j) b_{j, k} c_{j+k}=\sum_{r=n}^{n-1+h(n)} c_{r} \sum_{k=s}^{h(n)}(k+n-r) b_{r-k, k}
$$

with $s=\max \left(n_{0}, r-n+1\right)$. Since $r \geqslant n$,

$$
\sum_{k=s}^{h(n)}(k+n-r) b_{r-k, k} \leqslant \sum_{k=n_{0}}^{h(r)} k b_{r-k, k} \leqslant K r
$$

and (3.13) follows.

Theorem 3.6. Assume (H1) and $(\mathrm{H} 2)$, and let $c$ be a solution of (1.1) on $[0, T)$ with $\rho_{0}=\sum_{j=1}^{\infty} j c_{j}(0)$. Then

$$
\sum_{j=1}^{\infty} j c_{j}(t)=\rho_{0} \quad \text { for all } \quad t \in[0, T)
$$

Proof. By (3.2), to prove the result, we have to show that

$$
\lim _{n \rightarrow \infty} \int_{0}^{t} \sum_{j=1}^{n-1} j \sum_{k=n-j}^{\infty} W_{j, k}(c(s)) d s=0
$$

Set $T=\{(j, k): j+k \geqslant n, k \leqslant h(n), j \leqslant n-1\}$. By (3.11) it is sufficient to show that

$$
\lim _{n \rightarrow \infty} \int_{0}^{t} \sum_{T} j W_{j, k}(c(s)) d s=0
$$

By (3.8) and (3.12),

$$
\lim _{n \rightarrow \infty} n \int_{0}^{t} \sum_{j=1}^{n-1} \sum_{k=n-j}^{n-1} W_{j, k}(c(s)) d s=0
$$


Using the symmetry of $W_{j, k}$ and (3.12) with $n$ replaced by $h(n)$, it follows that

$$
\lim _{n \rightarrow \infty} n \int_{0}^{t} \sum_{T} W_{j, k}(c(s)) d s=0
$$

Finally, by writing $j W_{j, k}=(j-n) W_{j, k}+n W_{j, k}$ and using (3.13) and (3.22), we prove (3.20).

For future applications, it is useful to generalize (3.2).

Theorem 3.7. Assume (H1) and (H2). Let $\left(g_{j}\right)$ be a sequence with $\left|g_{j}-g_{k}\right| \leqslant K_{1}|j-k|$ for all $j, k$, where $K_{1}$ is a constant. Then if $c$ is a solution of $(1.1)$ on $[0, T)$, for all $t \in[0, T)$,

$$
\lim _{n \rightarrow \infty} \int_{0}^{t} \sum_{j=1}^{n} g_{j} \sum_{k=n-j+1}^{\infty} W_{j, k}(c(s)) d s=0
$$

The above result is proved in exactly the same way as Theorem 3.6.

\section{UNIQUENESS}

As noted in the introduction, in general, solutions of (1.1) need not be unique (see also Section 6). However, by imposing growth conditions on the kinetic coefficients, we are able to prove uniqueness.

Theorem 4.1. Let $K>0$ and $0 \leqslant \alpha \leqslant 1 / 2$ and assume the following:

(i) $a_{j, k} \leqslant K(j k)^{\alpha}$ for all $j, k$.

(ii) $\sum_{j=1}^{h(r)} j^{1-\alpha} b_{r-j, j} \leqslant K r^{1-\alpha}$ for all $r \geqslant 2$.

Let $c_{0} \in X^{+}$and $T>0$. Then there is exactly one solution $c$ of (1.1) on $[0, T)$ satisfying $c(0)=c_{0}$.

Proof. Let $c, d$ be two solutions of $(1.1)$ on $[0, T)$ satisfying $c(0)=$ $d(0)=c_{0}$ and set $x=c-d$. Let $\beta=1-\alpha$ and

$$
\vartheta(t)=\sum_{j=1}^{\infty} j^{\beta}\left|x_{j}(t)\right|
$$

We show that

$$
\vartheta(t) \leqslant \text { const. } \int_{0}^{t} \vartheta(s) d s, \quad t \in[0, T)
$$

so that, by Gronwall's lemma, $\vartheta(t)=0$ and $c=d$. 
For $\lambda \in \mathbb{R}$, define $\operatorname{sgn} \lambda$ to equal 1,0 , or -1 according as $\lambda>0, \lambda=0$, or $\lambda<0$. Note that if $\varphi(\cdot)$ is an absolutely continuous function of $t$, then so is $t \mapsto|\varphi(t)|$, and

$$
\frac{d}{d t}|\varphi(t)|=\operatorname{sgn} \varphi(t) \frac{d \varphi}{d t}(t) \text { a.e. }
$$

For $t \in[0, T)$, it thus follows from the same calculation leading to Lemma 3.1 that

$$
\sum_{j=1}^{n} j^{\beta}\left|x_{j}(t)\right|=\int_{0}^{t}\left[U_{n}(s)+V_{n}(s)\right] d s
$$

where

$$
\begin{gathered}
U_{n}=\frac{1}{2} \sum_{j+k \leqslant n}\left(g_{j+k}-g_{j}-g_{k}\right) M_{j, k}, \quad V_{n}=-\sum_{j=1}^{n} g_{j} \sum_{k=n-j+1}^{\infty} M_{j, k} \\
g_{j}=j^{\beta} \operatorname{sgn}\left(x_{j}\right) \\
M_{j, k}=W_{j, k}(c)-W_{j, k}(d)=a_{j, k}\left(c_{j} x_{k}+d_{k} x_{j}\right)-b_{j, k} x_{j+k}
\end{gathered}
$$

We first estimate $U_{n}$. Now

$$
\begin{aligned}
& {\left[(j+k)^{\beta} \operatorname{sgn}\left(x_{j+k}\right)-j^{\beta} \operatorname{sgn}\left(x_{j}\right)-k^{\beta} \operatorname{sgn}\left(x_{k}\right)\right] x_{k}} \\
& \quad=\left[(j+k)^{\beta} \operatorname{sgn}\left(x_{j+k} x_{k}\right)-j^{\beta} \operatorname{sgn}\left(x_{k} x_{j}\right)-k^{\beta}\right]\left|x_{k}\right| \\
& \quad \leqslant\left[(j+k)^{\beta}+j^{\beta}-k^{\beta}\right]\left|x_{k}\right| \leqslant 2 j^{\beta}\left|x_{k}\right|
\end{aligned}
$$

Thus,

$$
\begin{aligned}
& \frac{1}{2} \sum_{j+k \leqslant n}\left(g_{j+k}-g_{j}-g_{k}\right) a_{j, k} c_{j}\left|x_{k}\right| \\
& \quad \leqslant K \sum_{j+k \leqslant n} j k^{\alpha} c_{j}\left|x_{k}\right| \\
& \quad \leqslant K \sum_{j=1}^{\infty} j c_{j} \sum_{k=1}^{\infty} k^{\alpha}\left|x_{k}\right| \leqslant \text { const. } \vartheta
\end{aligned}
$$

since $\alpha \leqslant 1-\alpha$. We get a similar estimate for the terms involving $d_{k} x_{j}$. To estimate the fragmentation terms in $U_{n}$, note that

$$
\begin{aligned}
& -\left[(j+k)^{\beta} \operatorname{sgn}\left(x_{j+k}\right)-j^{\beta} \operatorname{sgn}\left(x_{j}\right)-k^{\beta} \operatorname{sgn}\left(x_{k}\right)\right] x_{j+k} \\
& \quad \leqslant\left[j^{\beta}+k^{\beta}-(j+k)^{\beta}\right]\left|x_{j+k}\right|
\end{aligned}
$$


Thus,

$$
\begin{aligned}
& -\sum_{j+k \leqslant n}\left(g_{j+k}-g_{j}-g_{k}\right) b_{j, k} x_{j+k} \\
& \quad \leqslant \sum_{j+k \leqslant n}\left[j^{\beta}+k^{\beta}-(j+k)^{\beta}\right] b_{j, k}\left|x_{j+k}\right| \\
& \quad \leqslant \sum_{r=2}^{n}\left|x_{r}\right| \sum_{j=1}^{r-1} \alpha_{r-j, j} b_{r-j, j}
\end{aligned}
$$

with $\alpha_{r-j, j}=(r-j)^{\beta}+j^{\beta}-r^{\beta} \leqslant j^{\beta}$. Also,

$$
\sum_{j=1}^{r-1} \alpha_{r-j, j} b_{r-j, j} \leqslant 2 \sum_{j=1}^{h(r)} \alpha_{r-j, j} b_{r-j, j} \leqslant 2 \sum_{j=1}^{h(r)} j^{\beta} b_{r-j, j} \leqslant 2 K r^{\beta}
$$

so that, by (4.4),

$$
-\sum_{j+k \leqslant n}\left(g_{j+k}-g_{j}-g_{k}\right) b_{j, k} c_{j+k} \leqslant \text { const. } \vartheta
$$

Combining (4.3), (4.5), for all $n$,

$$
\int_{0}^{t} U_{n}(s) d s \leqslant \text { const. } \int_{0}^{t} \vartheta(s) d s
$$

Next we show that

$$
\lim _{n \rightarrow \infty} \int_{0}^{t} V_{n}(s) d s=0
$$

It is easy to check that the assumptions on the kinetic coefficients imply that (H1) and (H2) hold. Thus, from (3.11),

$$
\begin{aligned}
& \lim _{n \rightarrow \infty} \int_{0}^{t}\left|\sum_{S} g_{j} M_{j, k}\right| d s \\
& \quad \leqslant \lim _{n \rightarrow \infty} \int_{0}^{t} \sum_{S} j\left[\left|W_{j, k}(c)\right|+\left|W_{j, k}(d)\right|\right] d s=0
\end{aligned}
$$

where $S=\{(j, k): k \geqslant h(n), 1 \leqslant j \leqslant n\}$. Hence, to prove $(4.7)$, it suffices to show that

$$
\lim _{n \rightarrow \infty} \int_{0}^{t} \sum_{S^{\prime}} g_{j} M_{j, k} d s=0
$$


where $S^{\prime}=\{(j, k): j+k \geqslant n, k \leqslant h(n), h(n) \leqslant j \leqslant n\}$. Now

$$
\sum_{S^{\prime}} j^{\beta} a_{j, k} c_{j} c_{k}=\sum_{j=h(n)}^{n-1} \sum_{k=n-j}^{h(n)} a_{j, k} c_{j} c_{k} \leqslant K \sum_{j=h(n)}^{n} j c_{j} \sum_{k=1}^{\infty} k^{\alpha} c_{k}
$$

Hence,

$$
\lim _{n \rightarrow \infty} \int_{0}^{t} \sum_{S^{\prime}} j^{\beta} a_{j, k} c_{j} c_{k} d s=0
$$

Applying Theorem 3.7 with $g_{j}=j^{\beta}$ and using (4.8) gives

$$
\lim _{n \rightarrow \infty} \int_{0}^{t} \sum_{S^{\prime}} j^{\beta} W_{j, k}(c(s)) d s=0
$$

Combining (4.10) and (4.11), we deduce that

$$
\lim _{n \rightarrow \infty} \int_{0}^{t} \sum_{S^{\prime}} j^{\beta}\left|W_{j, k}(c(s))\right| d s=0
$$

Then (4.9) follows immediately, completing the proof.

We can also prove uniqueness of density-conserving solutions at the expense of making very strong assumptions about the coagulation coefficients.

Theorem 4.2. Suppose that $a_{j, k} \leqslant K$ for all $j, k$. Let $c_{0} \in X^{+}$and $T>0$. Then there is at most one density-conserving solution $c$ of (1.1) on $[0, T)$ with $c(0)=c_{0}$.

Proof. The proof is very similar to that given for the previous result, so we only give the main steps. Let $c, d$ be density-conserving solutions with $c(0)=d(0)=c_{0}$ and let $\psi(t)=\sum_{j=1}^{\infty} j\left|x_{j}(t)\right|$. From the proof of the previous result, (4.2) holds with $\beta=1$. Using $a_{j, k} \leqslant K$, it is easy to show that if $g_{j}=j \operatorname{sgn}\left(x_{j}\right)$, then

$$
\sum_{j+k \leqslant n}\left(g_{j+k}-g_{j}-g_{k}\right)\left(c_{j} x_{k}+x_{j} d_{k}\right) a_{j_{*} k} \leqslant \text { const. } \psi
$$

Also,

$$
\begin{gathered}
-\left[(j+k) \operatorname{sgn}\left(x_{j+k}\right)-j \operatorname{sgn}\left(x_{j}\right)-k \operatorname{sgn}\left(x_{k}\right)\right] x_{j+k} \\
\leqslant[j+k-(j+k)]\left|x_{j+k}\right|=0
\end{gathered}
$$

Thus,

$$
\int_{0}^{t} U_{n}(s) d s \leqslant \text { const. } \psi(t)
$$


Finally, we have to show that

$$
\lim _{n \rightarrow \infty} \int_{0}^{t} \sum_{U} g_{j} M_{j, k} d s=0
$$

where $U=\{(j, k): j \leqslant n, j+k>n\}$. Since $c$ and $d$ conserve density, by applying Lemma 3.1 to $\sum_{j=1}^{n} j\left(c_{j}-d_{j}\right)$, we obtain

$$
\lim _{n \rightarrow \infty} \int_{0}^{l} \sum_{U} j M_{j, k} d s=0
$$

where $M_{j, k}=W_{j, k}(c)-W_{j, k}(d)$. Using the bounds on $a_{j, k}$, it is easy to show that

$$
\lim _{n \rightarrow \infty} \int_{0}^{l} \sum_{U} j a_{j, k} c_{j} c_{k} d s=0
$$

Combining (4.14) and (4.15) proves (4.13) and the result follows.

\section{CONTINUOUS DEPENDENCE}

Solutions are not unique in general, so by gluing together various solutions, we can demonstrate unpleasant analytic phenomena. For example, let $a_{j, k}=0, b_{j, k}=1$. Then it is shown in Example 6.2(b) that there is a solution $\bar{c}$ of $(1.1)$ with $\bar{c}(0)=0$ and density $\sum_{r=1}^{\infty} r \bar{c}_{r}(t)=1$ for $t>0$. Let $c$ be the solution of (1.1) defined by

$$
c(t)= \begin{cases}0, & 0<t<T \\ \bar{c}(t-T), & t \geqslant T\end{cases}
$$

Then $c$ is a solution on $[0, \infty)$ with $\sum_{r=1}^{\infty} r c_{r}(t)$ discontinuous at $t=T$.

To obtain good analytic information on solutions, we need to filter out these spurious solutions. Thus, in what follows we usually assume the conditions of density conservation.

Theorem 5.1. Let $c$ be a solution of (1.1) on $[0, T), T \leqslant \infty$. Assume that $c$ conserves density on $[0, T)$. Then $c:[0, T) \rightarrow X^{+}$is continuous, and the series $\sum_{r=1}^{\infty} r c_{r}(t)$ is uniformly convergent on compact intervals of $[0, T)$.

Proof. This follows from applying Dini's theorem to $\sum_{r=1}^{n} r c_{r}(t)$.

Theorem 5.2. Let $c$ be a solution of (1.1) on $[0, T), T \leqslant \infty$. Assume $a_{j, k} \leqslant K(j+k), b_{j, k} \leqslant K j k$, and that $c$ conserves density on $[0, T)$. Then each $c_{j}$ is continuously differentiable on $[0, T)$. 
Proof. The growth assumptions on the kinetic coefficients and Theorem 5.1 imply that the right-hand side of (1.1) is continuous in $t$, and the result follows.

Definition. A generalized flow $G$ on a metric space $Y$ is a family of continuous mappings $\phi:[0, \infty) \rightarrow Y$ with the following properties:

(i) If $\phi \in G$ and $\tau \geqslant 0$, then $\phi_{\tau} \in G$, where

$$
\phi_{\tau}(t) \stackrel{\text { def }}{=} \phi(t+\tau), \quad t \in[0, \infty)
$$

(ii) If $y \in Y$, there exists at least one $\phi \in G$ with $\phi(0)=y$.

(iii) If $\phi_{j} \in G$ with $\phi_{j}(0)$ convergent in $Y$ as $j \rightarrow \infty$, then there exist a subsequence $\phi_{j_{k}}$ of $\phi_{j}$ and an element $\phi \in G$ such that $\phi_{j_{k}}(t) \rightarrow \phi(t)$ in $Y$ uniformly for $t$ in compact intervals of $[0, \infty)$.

The following, which are analogues of results in Section 2, will be used in the proof of the upper semicontinuity property (iii) for the set of densityconserving solutions of (1.1).

Lemma 5.3. Assume $a_{j, k} \leqslant K(j+k)$ and let $c$ be a density-conserving solution of $(1.1)$ on $[0, \infty)$. Then for a.e. $t \geqslant 0$,

$$
\begin{aligned}
& \dot{x}_{m}=\frac{1}{2} \sum_{Z_{2}}(j+k) W_{j, k}(c)+\sum_{Z_{3}} j W_{j, k}(c) \\
& \dot{q}_{m}=\frac{1}{2} \sum_{Z_{1}} \mu_{j, k} W_{j, k}(c)+\frac{1}{2} \sum_{Z_{2}}(j+k) W_{j, k}(c)+\sum_{Z_{3}} \lambda_{j, k} W_{j, k}(c)
\end{aligned}
$$

and

$$
\frac{d}{d t}\left[e^{-t}\left(x_{m}+2 K m \rho^{2}\right)\right] \leqslant 0
$$

where

$$
\begin{gathered}
x_{m}=\sum_{r=m}^{\infty} r c_{r}, \quad q_{m}=\sum_{r=m}^{2 m} r c_{r}+2 m \sum_{r=2 m+1}^{\infty} c_{r} \\
Z_{1}=\{(j, k): j, k \geqslant m\}, \quad Z_{2}=\{(j, k): j+k \geqslant m, j, k<m\} \\
Z_{3}=\{(j, k): 1 \leqslant j \leqslant m-1, k \geqslant m\}, \quad \rho=\sum_{r=1}^{\infty} r c_{r}(0)
\end{gathered}
$$

and $\lambda_{j, k}, \mu_{j, k}$ are as defined in the proof of Theorem 2.4. 
Proof. Applying Lemma 3.1 to $x_{m}=\rho-\sum_{r=1}^{m-1} r c_{r}(t)$ gives $\dot{x}_{m}=$ $\sum_{z} j W_{j, k}$, where $Z=\{(j, k): 1 \leqslant j \leqslant m-1, j+k \geqslant m\}$. Since $Z$ is the disjoint union of $Z_{2}$ and $Z_{3}$,

$$
\dot{x}_{m}=\sum_{Z_{2}} j W_{j, k}+\sum_{Z_{3}} j W_{j, k}
$$

Then (5.1) follows from the symmetry of $Z_{2}$. Using Lemma 3.2 and Proposition 3.3, we obtain a formula for the derivative of the second term in $q_{m}$, and (5.2) follows by combining this with the derivative of the first term in $q_{m}$. Finally,

$$
\dot{x}_{m}=\sum_{Z} j W_{j, k} \leqslant K m \sum_{Z}(j+k) c_{j} c_{k} \leqslant 2 K m \rho^{2}
$$

from which we get (5.3).

Theorem 5.4. Assume $a_{j, k} \leqslant K(j+k)$ for all $j, k$. Let $G$ denote the set of all density-conserving solutions $c$ of $(1.1)$ on $[0, \infty)$. Then $G$ is a generalized flow on the closed metric subspace $X^{+}$of $X$.

Proof. By Theorem 5.1, if $c \in G$, then $c:[0, T) \rightarrow X^{+}$is continuous. The semigroup property (i) follows from (2.1), while property (ii) follows from Theorem 2.4. To check property (iii), let $c^{(n)}$ be a sequence of solutions of $(1.1)$ on $[0, \infty)$ with each $c^{(n)} \in G$ and $c^{(n)}(0) \rightarrow c_{0}$ in $X$ as $n \rightarrow \infty$. We repeat the proofs of Theorem 2.4 and Corollary 2.6 with $c^{(n)}$ playing the role of the approximating solutions. Since the details are very similar, we only outline the changes required. Set

$$
\vartheta_{m}^{n}(t)=e^{-t}\left[x_{m}^{n}(t)+2 K m\left(\rho^{n}\right)^{2}\right]
$$

where $\rho^{n}$ is the density of $c^{n}$ and $x_{m}^{n}=\sum_{r=m}^{\infty} r c_{r}^{n}$. Lemma 5.3 ensures that we can apply Helly's theorem to $\vartheta_{m}^{n}$. By using Lemma 5.3, we derive the analogue of $(2.10)$ :

$$
x_{m}^{n}(t)=x_{m}^{n}(0)+q_{m}^{n}(t)-q_{m}^{n}(0)+\int_{0}^{t}\left[\sum_{Z_{3}}\left(j-\lambda_{j, k}\right)-\sum_{Z_{1}} \mu_{j, k}\right] W_{j, k}\left(c^{n}(s)\right) d s
$$

where

$$
q_{m}^{n}=\sum_{r=m}^{2 m} r c_{r}^{n}+2 m \sum_{r=2 m+1}^{\infty} c_{r}^{n}
$$

Control of $x_{m}^{n}(0)$ follows from the strong convergence of $c^{n}(0)$. Finally,

$$
\sum_{Z_{3}} j a_{j, k} c_{j}^{n} c_{k}^{n} \leqslant K \sum_{j=1}^{m-1} j c_{j}^{n} \sum_{k=m}^{\infty}(j+k) c_{k}^{n} \leqslant 2 K\left\|c^{n}\right\| x_{m}^{n}
$$

with a similar estimate for the sum over $Z_{1}$. 
We also consider the continuous dependence of solutions with respect to weak* convergence in $X$. Recall that $y^{j}$ converges in the weak* sense to $y \in X$ (symbolically $y_{r}^{j} \stackrel{*}{\sim} y_{r}$ ) if

(i) $\sup _{j}\left\|y^{j}\right\|<\infty$.

(ii) $y_{r}^{j} \rightarrow y_{r}$ as $j \rightarrow \infty$ for each $r=1,2, \ldots$.

For $\rho>0$ let $B_{\rho}=\{y \in X:\|y\| \leqslant \rho\}$. Then $\left(B_{\rho}, d\right)$ is a metric space with metric $d(y, z)=\sum_{r=1}^{\infty}\left|y_{r}-z_{r}\right|$. Clearly, a sequence $\left\{y^{j}\right\} \subset B_{\rho}$ converges in the weak* sense to $y \in X$ if and only if $y \in B_{r}$ and $d\left(y^{j}, y\right) \rightarrow 0$ as $j \rightarrow \infty$. For $\rho>0$ set $B_{\rho}^{+}=B_{\rho} \cap X^{+}$; then $B_{\rho}^{+}$is a closed metric subspace of $B_{\rho}$.

Theorem 5.5. Let $g_{j} \geqslant 0$ with $g_{j}=o(j)$ as $j \rightarrow \infty$. Assume that

$$
a_{j, k} \leqslant g_{j}+g_{k}, \quad b_{j, k} \leqslant g_{j} g_{k}
$$

for all $j, k$. For $\rho>0$ let $G_{\rho}$ denote the set of all solutions $c$ of (1.1) on $[0, \infty)$ with $c(0) \in B_{\rho}^{+}$. Then $G_{\rho}$ is a generalized flow on $B_{\rho}^{+}$.

The proof of the above theorem is a simple application of the ArzelaAscoli theorem, so we omit it.

\section{EXAMPLES}

In this section we give a number of results which highlight the role of the assumptions on the kinetic coefficients. We begin with nonexistence results. Let $b_{i, j}=0$ and $a_{i, j}=r_{i}+r_{j}$. A formal calculation in ref. 8 showed that solutions with time-dependent densities do not exist for these kernels (cf. Theorem 3.6 for a rigorous proof of this). For the case $r_{j}=j^{\alpha}, \alpha>1$, it is formally argued in ref. 8 that solutions do not exist globally in time. We give two rigorous nonexistence results for kernels of this type. The first shows that for any initial data, the corresponding solution only exists for a finite time; the second shows that for a class of initial data, there are no solutions even on a short time interval.

Theorem 6.1. Let $b_{j, k}=0$ and $a_{j, k}=r_{j}+r_{k}+\alpha_{j, k}$, where $0 \leqslant \alpha_{j, k} \leqslant$ $K_{1}(j+k)$ and $r_{j} \geqslant 0$.

(i) Suppose $r_{j} \geqslant K_{2} j^{\alpha}$, where $K_{2}>0$ and $\alpha>1$. If $c$ is a solution of (1.1) on $[0, T)$ with $c(0) \neq 0$, then $T<\infty$.

(ii) Suppose $j^{-1} r_{j} \rightarrow \infty$ as $j \rightarrow \infty$. Let $c_{0}=\left(c_{0 j}\right) \in X^{+}$be such that $\exp \left(\gamma_{m} \delta\right) \sum_{j=m+1}^{\infty} j c_{0 j}$ does not tend to zero as $m \rightarrow \infty$ for all $\delta>0$, where $\gamma_{m} \stackrel{\text { def }}{=} \min _{j \geqslant m} j^{-1} r_{j}$. Then there is no solution $c$ of (1.1), defined on any interval $[0, T), T>0$, and with initial data $c(0)=c_{0}$. 
Proof. (i) Suppose for contradiction that $c$ is a solution of (1.1) on $[0, \infty)$ with $c(0) \neq 0$. Set $h(t)=\sum_{j=1}^{\infty} c_{j}(t)$. By Lemma 3.3, Proposition 3.4, and (3.5), $h$ is absolutely continuous and

$$
h(t)-h(0)=-\int_{0}^{t} Q(s) d s
$$

where

$$
Q=\frac{1}{2} \sum_{j, k=1}^{\infty} a_{j, k} c_{j} c_{k} \geqslant\left(\sum_{j=1}^{\infty} r_{j} c_{j}\right)\left(\sum_{k=1}^{\infty} c_{k}\right)
$$

By Hölder's inequality,

$$
\left(\sum_{j=1}^{\infty} j c_{j}\right)^{\alpha}\left(\sum_{j=1}^{\infty} c_{j}\right)^{1-\alpha} \leqslant \sum_{j=1}^{\infty} j^{\alpha} c_{j}
$$

Using this and the conservation of density (Theorem 3.6) in (6.2) gives $Q \geqslant K_{3} h^{2-\alpha}$, where $K_{3}>0$ is a constant. Hence, from (6.1),

$$
\dot{h}(t) \leqslant-K_{3} h(t)^{2-\alpha} \quad \text { a.e. } t \in[0, \infty)
$$

It follows easily from this that $h\left(t_{0}\right)=0$ for some $t_{0}>0$, which contradicts the positiveness of the density.

(ii) Let $c$ be a solution on $[0, T)$ with $c(0)=c_{0}$, where $T<\infty$. Set $u_{m}(t)=\sum_{j=m+1}^{\infty} j c_{j}(t)$ for $t \in[0, T)$. From Theorem 3.6 and Lemma 3.1

$$
u_{m}(t)-u_{m}(0)=\int_{0}^{t} p_{m}(s) d s
$$

where

$$
p_{m}=\sum_{j=1}^{m} \sum_{k=m-j+1}^{\infty} j a_{j, k} c_{j} c_{k} \geqslant \sum_{j=1}^{m} j c_{j} \sum_{k=m+1}^{\infty} r_{k} c_{k}
$$

By Theorem 5.1, there exists $M>0$ such that $\sum_{j=1}^{m} j c_{j}(t) \geqslant \rho / 2$ for all $m>M, t \in[0, T)$ where $\rho$ is the density. Thus, for $m>M, p_{m} \geqslant(\rho / 2) \gamma_{m} u_{m}$, so that

$$
u_{m}(t)-u_{m}(0) \geqslant(\rho / 2) \gamma_{m} \int_{0}^{t} u_{m}(s) d s
$$

Hence $u_{m}(t) \geqslant \exp \left[(\rho / 2) \gamma_{m} t\right] u_{m}(0)$, which contradicts $u_{m}(t) \rightarrow 0$ as $m \rightarrow \infty$. 
There are always $c_{0} \in X^{+}$satisfying condition (ii) of the above theorem (see ref. 2, p. 670).

Next we give an example which shows both that the density need not be conserved by all solutions of (1.1) and that in general we do not have continuous dependence of solutions with respect to weak* convergence for the set of density-conserving solutions.

Example 6.2. Let $a_{i, j}=0, b_{i, j}=(i+j)^{\beta}$, so that (1.1) takes the form

$$
\dot{c}_{j}=\sum_{k=j+1}^{\infty} k^{\beta} c_{k}-\frac{1}{2} c_{j}(j-1) j^{\beta}
$$

(a) For $\lambda>0, \beta>-1$, define a sequence $y_{j}(\lambda)$ by $y_{1}(\lambda)=1$ and

$$
\alpha_{j} y_{j}-\alpha_{j+1} y_{j+1}=(j+1)^{\beta} y_{j+1}, \quad j \geqslant 1
$$

where $\alpha_{j}=\lambda+\frac{1}{2}(j-1) j^{\beta}$. Define $c_{j}(t)=e^{\lambda t} y_{j}(\lambda)$. Writing $y_{j}=j^{-(\beta+3)} z_{j}$, it is not hard to prove that $z_{j+1}=\left(1+\delta_{j}\right) z_{j}$, where $\delta_{j}=O\left(j^{-\gamma}\right)$ and $\gamma=\min (3,2+\beta)$. It follows that $0 \leqslant y_{j}(\lambda) \leqslant$ const. $j^{-(\beta+3)}$ for all $j$. In particular, $\left(y_{j}(\lambda)\right) \in X^{+}$and

$$
\sum_{k=j+1}^{\infty} k^{\beta} c_{k}=\sum_{k=j}^{\infty}\left(\alpha_{j} c_{j}-\alpha_{j+1} c_{j+1}\right)=\alpha_{j} c_{j}
$$

so that $c=\left(c_{j}\right)$ satisfies (6.3). Thus, for $\beta>-1$ we have a solution $c$ as defined in Section 2 with density $K e^{\lambda t}, K$ a constant. If, on the other hand, $\beta \leqslant-1$, then from Theorem 3.6 we see that any solution of (6.3) with finite density must conserve density.

(b) Consider the special case $\beta=0$, i.e., $a_{i, j}=0, b_{i, j}=1$, so that (6.3) becomes

$$
\dot{c}_{j}=\sum_{k=j+1}^{\infty} c_{k}-\frac{1}{2}(j-1) c_{j}
$$

For $r=1,2, \ldots$, let $c_{0}^{r} \in X^{+}$be given by $c_{0}^{r}=\left(r^{-1} \delta_{r, j}\right)$, so that $\left\|c_{0}^{r}\right\|=1$ for all $r$ and $c_{0}^{r}$ converges in the weak* sense to the zero sequence as $r \rightarrow \infty$. The unique density-conserving solution $c^{r}(t)$ of (5.4) with initial data $c_{0}^{r}$ is given by

$$
\begin{array}{cc}
c_{j}^{r}(t)=r^{-1}\left(e^{-t / 2}\right)^{j-1}\left[2\left(1-e^{-t / 2}\right)+\left(1-e^{-t / 2}\right)^{2}(r-j-1)\right] & \text { if } j<r \\
c_{j}^{j}(t) & =j^{-1}\left(e^{-t / 2}\right)^{j-1} \\
c_{j}^{r}(t) & =0 \quad \text { for } j>r
\end{array}
$$


Then, as $r \rightarrow \infty, c^{r}{ }^{*} \bar{c}=\left(\bar{c}_{j}\right)$, where $\bar{c}_{j}(t)=\left(e^{-t / 2}\right)^{j-1}\left(1-e^{-t / 2}\right)^{2}$. It is easy to check that $\bar{c}$ is a solution of $(6.5)$ with $\bar{c}(0)=0$ and $\|\bar{c}(t)\|=1$ for all $t>0$.

By taking linear combinations of the solutions in Example 6.2, it is seen that solutions of (6.3) are nonunique for any initial data in the case $\beta>-1$. Clearly, these are nonphysical solutions. Theorems 2.9 and 4.2 show that if we define an admissible solution of (1.1) to be a solution of (1.1) which is a limit of the truncated system (2.1), then admissible solutions are unique and conserve density.

Example 6.3. If $a_{j, k}=j+k, b_{j, k}=0$, then the conclusion of Theorem 5.5 is false. To see this, we note that by using Theorems 5.1 and 5.2 , we can differentiate the relation in (3.5) to get

$$
\dot{M}(t)=-\rho M(t)
$$

where

$$
M(t)=\sum_{j=1}^{\infty} c_{j}(t), \quad \rho=\sum_{j=1}^{\infty} j c_{j}(t)
$$

Also,

$$
\dot{c}_{1}(t)=-[\rho+M(t)] c_{1}(t)
$$

Solving (6.6)-(6.7) gives

$$
c_{1}(t)=c_{1}(0) \exp \left\{\rho^{-1} M(0)[\exp (-\rho t)-1]-\rho t\right\}
$$

Therefore, if $c^{r}(0) \stackrel{*}{\rightarrow} c_{0}$ with

$$
\lim _{r \rightarrow \infty} \sum_{j=1}^{\infty} j c_{j}^{r}(0)=\bar{\rho}>\rho=\sum_{j=1}^{\infty} j c_{0 j}
$$

and $c \neq 0$, then

$$
\lim _{r \rightarrow \infty} \sum_{j=1}^{\infty} c_{j}^{r}(0)=\sum_{j=1}^{\infty} c_{0 j} \stackrel{\text { def }}{=} \alpha
$$

and

$$
\begin{aligned}
\lim _{r \rightarrow \infty} c_{1}^{r}(t) & =c_{01} \exp \left\{(\bar{\rho})^{-1} \alpha[\exp (-\bar{\rho} t)-1]-\bar{\rho} t\right\} \\
& \not \neq c_{01} \exp \left\{\rho^{-1} \alpha[\exp (-\rho t)-1]-\rho t\right\}
\end{aligned}
$$


For applications to phase transitions in a quenched binary alloy, one set of conditions suggested by $O$. Penrose on the coagulation and fragmentation rates is that $a_{j, k}=O\left(j^{1 / 3}+k^{1 / 3}\right)$ and that $b_{j, k}=a_{j, k} Q_{j} Q_{k}\left(Q_{j+k}\right)^{-1}$, where $Q_{j} \sim z_{s}^{-j} \exp \left(-\lambda j^{1 / 3}\right)$ with $\lambda, z_{s}$ positive constants. Note that in this case we may have $b_{j, k} \sim j^{1 / 3}$ for $j$ large and $k$ bounded, while for $j$ and $k$ large with $j-k$ bounded, $b_{j, k}$ is small. The physical motivation here is that surface area considerations show that it is unlikely that a large cluster of size $j+k$ will split into two large clusters of size $j$ and $k$ (and hence increase the surface energy by a large amount).

Proposition 6.4. (a) The hypotheses of Theorem 3.6 (conservation of density) are satisfied if $a_{j, k} \leqslant K(j+k), \quad b_{j, k} \leqslant K(j+k)$ $\exp \left\{\lambda\left[(j+k)^{p}-j^{p}-k^{p}\right]\right\}$, where $K, \lambda>0$ and $0<p<1$.

(b) The hypotheses of Theorem 4.1 (uniqueness) are satisfied if $a_{j, k} \leqslant K(j k)^{\alpha}, b_{j, k} \leqslant K(j+k)^{1-\alpha} \exp \left\{\lambda\left[(j+k)^{p}-j^{p}-k^{p}\right]\right\}$, where $K, \lambda>0$, $0 \leqslant \alpha \leqslant 1 / 2,0<p<1$.

Proof. We need only check the conditions on $b_{j, k}$. Fix $B$ with $0<B<2-2^{p}$. Then it is easy to show that there exists $r_{0}$ such that for $1 \leqslant j \leqslant h(r)$ and $r \geqslant r_{0}$,

$$
r^{p}-(r-j)^{p}-j^{p} \leqslant-B j^{p}
$$

Thus, in case (a), for $r \geqslant r_{0}$ and $1 \leqslant j \leqslant h(r)$, using (6.8), we have that $b_{r-j, j} \leqslant K r \exp \left(-B j^{p}\right)$, with a similar inequality for case (b).

\section{REFERENCES}

1. M. Aizenman and T. A. Bak, Convergence to equilibrium in a system of reacting polymers, Commun. Math. Phys. 65:203-230 (1979).

2. J. M. Ball, J. Carr, and O. Penrose, The Becker-Döring cluster equations: Basic properties and asymptotic behaviour of solutions, Commun. Math. Phys. 104:657-692 (1986).

3. J. M. Ball and J. Carr, Asymptotic behaviour of solutions to the Becker-Döring equations for arbitrary initial data, Proc. R. Soc. Edinburgh 108A:109-116 (1988).

4. J. M. Ball and J. Carr, In preparation.

5. K. Binder, Theory for the dynamics of clusters. II. Critical diffusion in binary systems and the kinetics of phase separation, Phys. Rev. B 15:4425 4447 (1977).

6. P. G. J. van Dongen, Spatial fluctuations in reaction-limited aggregation, J. Stat. Phys. 54:221-271 (1989).

7. R. Drake, In Topics in Current Aerosol research, G. M. Hidy and J. R. Brock, eds. (Pergamon Press, Oxford, 1972).

8. E. M. Hendricks, M. H. Ernst, and R. M. Ziff, Coagulation equations with gelation, J. Stat. Phys. 31:519-563 (1983).

9. F. Leyvraz and H. R. Tschudi, Singularities in the kinetics of coagulation processes, J. Phys. A: Math. Gen. 14:3389-3405 (1981). 
10. F. Leyvraz and H. R. Tschudi, Critical kinetics near gelation, J. Phys. A: Math. Gen. 15:1951-1964 (1982).

11. J. B. McLeod, On an infinite set of non-linear differential equations, Q. J. Math. Ser. (2) 13:119-128 (1962).

12. O. Penrose, Metastable states for the Becker--Döring cluster equations, Commun. Math. Phys. 121:527-540 (1989).

13. G. E. H. Reuter and W. Ledermann, On the differential equations for the transitional probabilities of Markov processes with enumerably many states, Proc. Camb. Phil. Soc. 49:247-262 (1953).

14. M. Slemrod, Trend to equilibrium in the Becker-Döring cluster equations, Nonlinearity 2:429-443 (1989).

15. J. L. Spouge, An existence theorem for the discrete coagulation-fragmentation equations, Math. Proc. Camb. Phil. Soc. 96:351-357 (1984).

16. I. W. Stewart, A global existence theorem for the general coagulation-fragmentation equation with unbounded kernels, Math. Meth. Appl. Sci. 11:627-648 (1989).

17. W. H. White, A global existence theorem for Smoluchowski's coagulation equations, Proc. Am. Math. Soc. 80:273-276 (1980). 